\title{
DEFORMATION IN THE LARGE OF SOME COMPLEX MANIFOLDS, II
}

\author{
FABRIZIO CATANESE \\ PAOLA FREDIANI
}

\begin{abstract}
The compact complex manifolds considered in this article are principal torus bundles over a torus. We consider the Kodaira Spencer map of the complete Appell Humbert family (introduced by the first author in Part I) and are able to show that we obtain in this way a connected component of the space of complex structures each time that the base dimension is two, the fibre dimension is one, and a suitable topological condition is verified.
\end{abstract}

\section{INTRODUCTION}

One of the most interesting problems in the theory of compact complex manifolds is "Moduli theory", i.e., the study of the space of complex structures $\mathcal{C}(M)$ on a given oriented differentiable manifold $M$. Viewing these as integrable almost complex structures yields a description as an infinite dimensional space on which acts the infinite dimensional group $\operatorname{Diff}^{+}(M)$ of orientation preserving diffeomorphisms of $M$.

Moduli theory in a proper sense means studying in detail the quotient $\mathfrak{M}(M):=\mathcal{C}(M) / D i f f^{+}(M)$, and often this study is split into two parts.

First one studies the Teichmüller space of $M$, i.e., $\mathfrak{T}(M):=$ $\mathcal{C}(M) / \operatorname{Diff}^{0}(M)$, where $\operatorname{Diff}^{0}(M) \subset \operatorname{Diff}^{+}(M)$ is the connected component of the identity (its elements are called the diffeomorphisms isotopic to the identity). Then one may view the Moduli space $\mathfrak{M}(M)$ as the quotient of the Teichmüller space $\mathfrak{T}(M)$ by the Mapping class group $\mathcal{M a p}(M):=$ $\operatorname{Diff} f^{+}(M) / D i f f^{0}(M)$, hoping that this action turn out to be properly discontinuous.

Of course a preliminary question is the determination of the connected components of $\mathcal{C}(M)$, which are called the deformation classes in the large of the complex structures on $M$, and which we believe may be countably many (their number may be arbitrarily high, cf. Cat02, Cat04). Observe that the usual deformation theory addresses only the study of the small deformations, i.e., it describes the so called Kuranishi space $\mathfrak{B}(M)$ which is the germ of the Teichmüller space at the point corresponding to a given complex structure. Kuranishi's theorem ([Ku1], $\underline{\mathrm{Ku} 2}]$ ) shows that the Teichmüller space is a complex

\footnotetext{
${ }^{1}$ The present research took place in the framework of the Schwerpunkt "Globale Methode in der komplexen Geometrie", and of the PRIN 2003: "Spazi dei moduli e teoria di Lie" (MURST)
}

AMS Subject classification: 14D15, 32G08, 32G13, 32L05, 32Q57, 53C30, 53C56.

Date: May 18, 2005. 
space of locally finite dimension (its dimension may however be unbounded, cf. cor. 7.7 of [Cat04]).

A prototype test for all these desiderata is furnished by the example of the complex tori. These are parametrized by an open set $\mathcal{T}_{n}$ of the complex Grassmann Manifold $\operatorname{Gr}(n, 2 n)$, image of the open set of matrices

$\left\{\Omega \in \operatorname{Mat}(2 n, n ; \mathbb{C}) \mid(i)^{n} \operatorname{det}(\Omega \bar{\Omega})>0\right\}$.

This parametrization is very explicit: if we consider a fixed lattice $\Gamma \cong \mathbb{Z}^{2 n}$, to each matrix $\Omega$ as above we associate the subspace $V=(\Omega)\left(\mathbb{C}^{n}\right)$, so that $V \in G r(n, 2 n)$ and $\Gamma \otimes \mathbb{C} \cong V \oplus \bar{V}$.

Finally, to $\Omega$ we associate the torus $Y_{V}:=V / p_{V}(\Gamma), p_{V}: V \oplus \bar{V} \rightarrow V$ being the projection onto the first addendum.

Not only we obtain in this way a connected open set inducing all the small deformations (cf. [K-M71), but indeed, as it was shown in Cat02 (cf. also Cat04) a connected component of the Teichmüller space.

It was observed however by Kodaira and Spencer already in their first article ( K-S58, and volume II of Kodaira's collected works) that the mapping class group $S L(2 n, \mathbb{Z})$ does not act properly discontinuously on $\mathcal{T}_{n}$. This shows that for compact complex manifolds it is better to consider, rather than the Moduli space, the Teichmüller space, which can be obtained by glueing together several Kuranishi spaces.

Moreover, after some initial constructions by Blanchard and Calabi (cf. Bla53, Bla56], Cal58]) of non Kähler complex structures $X$ on manifolds diffeomorphic to a product $C \times T$, where $C$ is a compact complex curve and $T$ is a 2-dimensional complex torus, Sommese observed (Somm75]) that the space of complex structures on a six dimensional real torus is not connected.

These examples were then generalized in Cat02 Cat04 under the name of Blanchard-Calabi manifolds showing (corollary 7.8 of [Cat04]) that also the space of complex structures on the product of a curve $C$ of genus $g \geq 2$ with a four dimensional real torus is not connected.

On the positive side, however, our goal is to determine completely the connected components of spaces $\mathcal{C}(M)$ (the so called equivalence classes for "deformation in the large") for suitable differentiable manifolds.

A very interesting class of examples to consider is given by the quotients of an affine space $\mathbb{C}^{n}$ by a nilpotent or solvable group $\Pi$. One of the main tools in order to analyse this type of examples should be the cited result that every deformation in the large of a complex torus is a complex torus.

Since this ambitious program carries several big difficulties with it, we begin to address it in the simplest possible case, i.e., when we have a holomorphic torus bundle $f: X \rightarrow Y$ over a torus $Y$ and the group is 2-step nilpotent (the central group extension is given by $\Lambda:=\pi_{1}(F) \hookrightarrow \pi_{1}(X)=\Pi \rightarrow \pi_{1}(Y)=\Gamma$, classified by an alternating bilinear form $\left.A \in \Lambda^{2}(\Gamma)^{\vee} \otimes(\Lambda)\right)$.

We shall use the classification theory for principal holomorphic torus bundles over tori given in Theorem 6.8 of Cat04, where an explicit family is produced, described purely in terms of bilinear algebra, and called the complete AppellHumbert family of Torus Bundles. 
Roughly speaking, one looks for the subspaces $U \subset \Lambda \otimes \mathbb{C}, V \subset \Gamma \otimes \mathbb{C}$ such that, viewing $A$ as a real element of

$$
\Lambda^{2}(\Gamma \otimes \mathbb{R})^{\vee} \otimes(\Lambda \otimes \mathbb{R})=\Lambda^{2}(V \oplus \bar{V})^{\vee} \otimes(U \oplus \bar{U}),
$$

its component in $\Lambda^{2}(\bar{V})^{\vee} \otimes(U)$ is zero (one says then that the Riemann bilinear relations hold).

This theory bears close similarities with the theory of line bundles over tori, and allows for explicit descriptions of several holomorphic invariants (see theorems $6.11-6.12$ of [Cat04]).

The simplest way to explain the meaning of the Appell- Humbert family is as follows: one observes that the differentiable manifold $M$ underlying $X$ is simply the quotient of a contractible real Lie group $\Pi \otimes \mathbb{R}$ by a discrete subgroup $\Pi$. We consider now all the right invariant almost complex structures on $\Pi \otimes \mathbb{R}$, and we show that the Riemann bilinear relations are equivalent to the integrability of these almost complex structures.

In particular, explicit formulae can be given for this family of complex structures, and from these one derives for instance

Theorem 6.10 of Cat04] : The cokernel of $0 \rightarrow H^{0}\left(\Omega_{Y}^{1}\right) \rightarrow H^{0}\left(\Omega_{X}^{1}\right)$ is the subspace of $U^{\vee}$ which annihilates the image of the Hermitian part $B^{\prime \prime}$ of $A, B^{\prime \prime} \in\left[(V \otimes \bar{V})^{\vee} \otimes(U)\right]$, i.e., $\left\{u^{\vee} \in U^{\vee} \mid u^{\vee} \dashv B^{\prime \prime}=0 \in(V \otimes \bar{V})^{\vee}\right\}$.

It follows in particular that $X$ is parallelizable if and only if the Hermitian part of $A$ is zero.

We already observed that $X$, as a differentiable manifold, is the quotient of the real Lie group $\Pi \otimes \mathbb{R}$ by the discrete subgroup $\Pi$ : the point is that there are some complex structures where we can realize $X$ as the quotient of a complex Lie group by $\Pi$.

The most famous example is the Iwasawa 3-fold (cf. [K-M71], whose small deformations were analysed by Nakamura in Nak75, who thus showed that small deformations of a complex parallelizable manifold need not be parallelizable.

Our first result in this paper will be the following

Theorem 4.11 Let $f: X \rightarrow Y$ be a holomorphic principal bundle with base a complex torus $Y:=Y_{V}$ of dimension $m=2$, and fibre an elliptic curve $T:=T_{U}$.

Then the complete Appell Humbert family is versal at $X$ if either $B^{\prime} \neq 0$, or $B^{\prime}=0$ and $B(v, x)$ non degenerate in the first factor, or $X$ is parallelizable and $A \neq 0$.

The strong restriction on the dimensions $m=2, d=1$ is forced by the requirement that the complete Appell Humbert family have a smooth basis. This condition, combined with the surjectivity of the Kodaira Spencer map, ensures that all the small deformations are again principal holomorphic torus bundles.

The above result combines with the following

Theorem 6.17 of Cat04 Let $f: X \rightarrow Y$ be a holomorphic principal bundle with base a complex torus $Y_{V}$ of dimension $m$, and fibre an elliptic curve $T_{U}$. 
Assume moreover $\pi_{1}(X):=\Pi$ to be a nontrivial central extension

$$
1 \rightarrow \Lambda \rightarrow \Pi \rightarrow \Gamma \rightarrow 1
$$

classified by a cohomology class $\epsilon \neq 0 \in H^{2}(Y, \Lambda)$ whose associated bilinear form $A$ has an image of dimension $=2$.

Then every limit of manifolds in the complete Appell-Humbert family is again a holomorphic principal bundle $f^{\prime}: X^{\prime} \rightarrow Y^{\prime}$ with fibre an elliptic curve $T^{\prime}$, and thus occurs in the complete Appell-Humbert family .

and one shows that the class of holomorphic torus bundles with fibre dimension $d=1$, and base dimension $m=2$ form irreducible components in the Teichmüller space.

With a further assumption, however, we are able to produce many connected components of the Teichmüller space

Main Theorem 4.13 Consider the family of holomorphic principal torus bundles $f: X \rightarrow Y$ with base a complex torus $Y_{V}$ of dimension 2, and with fibre an elliptic curve $T_{U}$, corresponding to a bilinear form $A$ such that

1) $A$ is non degenerate and $\operatorname{Im} A$ has dimension 2

2) the associated pencil

$\mathbb{P}^{1} \cong \mathbb{P}\left((\Lambda \otimes \mathbb{R})^{\vee}\right) \hookrightarrow \mathbb{P}\left(\Lambda^{2}(\Gamma \otimes \mathbb{R})^{\vee}\right) \cong \mathbb{P}\left(\Lambda^{2}\left(\mathbb{R}^{4}\right)\right)$ intersects the Klein Pfaffian quadric in at least one real point.

Then this family forms a connected component of the Teichmüller space.

Indeed condition 2) is necessary: in fact there are deformations of the Iwasawa manifold for which the complete Appell Humbert family has not surjective Kodaira Spencer map.

Time reasons do not allow us to investigate fully the difficult question whether in this and similar cases the complete Appell Humbert family fails to yield an open set in the Teichmüller space.

\section{PRINCIPAl holomorPhic torus BUNDLES OVER TORI: GENERALITIES}

Throughout the paper, our set up will be the following: we have a holomorphic submersion between compact complex manifolds

$$
f: X \rightarrow Y
$$

such that the base $Y$ is a complex torus, and one fibre $F$ (whence all the fibres, by theorem 2.1 of [Cat04]) is also a complex torus.

We shall denote this situation by saying that $f$ is differentiably a torus bundle.

We let $n=\operatorname{dim} X, m=\operatorname{dim} Y, d=\operatorname{dim} F=n-m$.

In general ( $\mathrm{cf}$. [FG65]) $f$ is a holomorphic bundle if and only if all the fibres are biholomorphic.

This holds necessarily in the special case $d=1$, because the moduli space for 1-dimensional complex tori exists and is isomorphic to $\mathbb{C}$. 
Assume now that we have a holomorphic torus fibre bundle, thus we have (cf. Cat04, pages 271-273) the exact sequence

$$
0 \rightarrow \Omega_{Y}^{1} \rightarrow f_{*} \Omega_{X}^{1} \rightarrow f_{*} \Omega_{X \mid Y}^{1} \rightarrow 0 .
$$

and a principal holomorphic bundle if moreover $f_{*} \Omega_{X \mid Y}^{1}$ is holomorphically trivial.

Remark 2.1. In general (cf. e.g. [BPV84]) if $T$ is a complex torus, we have an exact sequence of complex Lie groups

$$
0 \rightarrow T \rightarrow \operatorname{Aut}(T) \rightarrow M \rightarrow 1
$$

where $M$ is discrete. Taking sheaves of germs of holomorphic maps with source $Y$ we get

$$
0 \rightarrow \mathcal{H}(T)_{Y} \rightarrow \mathcal{H}(\operatorname{Aut}(T))_{Y} \rightarrow M \rightarrow 1
$$

and the exact sequence

$$
0 \rightarrow H^{1}\left(Y, \mathcal{H}(T)_{Y}\right) \rightarrow H^{1}(Y, \mathcal{H}(\operatorname{Aut}(T)))_{Y} \rightarrow H^{1}(Y, M)
$$

(since holomorphic bundles with base $Y$ and fibre $T$ are classified by the cohomology group $H^{1}\left(Y, \mathcal{H}(A u t(T))_{Y}\right)$ ) determines the discrete obstruction for a holomorphic bundle to be a principal holomorphic bundle.

In the case of a principal holomorphic bundle we write $\pi_{1}(T) \cong \Lambda, \pi_{1}(Y) \cong \Gamma$ and the exact sequence

$$
\rightarrow H^{0}\left(\mathcal{H}(T)_{Y}\right) \rightarrow H^{1}(Y, \Lambda) \rightarrow H^{1}\left(Y, \mathcal{O}_{Y}^{d}\right) \rightarrow H^{1}\left(\mathcal{H}(T)_{Y}\right) \rightarrow^{c} \rightarrow H^{2}(Y, \Lambda)
$$

determines a cohomology class $\epsilon \in H^{2}(Y, \Lambda)$ which classifies the central extension

$$
1 \rightarrow \pi_{1}(T)=\Lambda \rightarrow \Pi:=\pi_{1}(X) \rightarrow \pi_{1}(Y)=\Gamma \rightarrow 1
$$

(it is central by the triviality of the monodromy automorphism).

Proposition 2.2. Let $f: X \rightarrow Y$ be a principal holomorphic torus bundle over a torus as above.

Then the universal covering of $X$ is isomorphic to $\mathbb{C}^{m+d}$ and $X$ is biholomorphic to a quotient $X \cong \mathbb{C}^{m+d} / \Pi$.

\section{Proof.}

Let $\pi: \mathbb{C}^{m} \rightarrow Y$ be the universal covering map and let us consider the pull back bundle $\pi^{*} X \rightarrow \mathbb{C}^{m}$ as in the following Cartesian diagram:

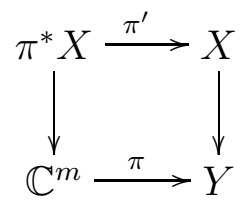

$\pi^{*} X \rightarrow \mathbb{C}^{m}$ is a principal holomorphic torus bundle on $\mathbb{C}^{m}$, therefore it is trivial. In fact principal holomorphic torus bundles on $\mathbb{C}^{m}$ are classified by $H^{1}\left(\mathbb{C}^{m}, \mathcal{H}(T)_{\mathbb{C}^{m}}\right)$, and we claim that $H^{1}\left(\mathbb{C}^{m}, \mathcal{H}(T)_{\mathbb{C}^{m}}\right)=0$. Let us in fact consider the exact sequence defining $T$,

$$
1 \rightarrow \Lambda \rightarrow U \rightarrow T \rightarrow 1
$$


Taking germs of holomorphic functions with source $\mathbb{C}^{m}$, we get

$$
1 \rightarrow \Lambda \rightarrow \mathcal{H}(U)_{\mathbb{C}^{m}} \rightarrow \mathcal{H}(T)_{\mathbb{C}^{m}} \rightarrow 1
$$

and the long exact cohomology sequence yields

$0=H^{1}\left(\mathbb{C}^{m}, \Lambda\right) \rightarrow H^{1}\left(\mathbb{C}^{m}, \mathcal{O}_{\mathbb{C}^{m}}^{d}\right)=0 \rightarrow H^{1}\left(\mathbb{C}^{m}, \mathcal{H}(T)_{\mathbb{C}^{m}}\right) \rightarrow H^{2}\left(\mathbb{C}^{m}, \Lambda\right)=0$

therefore $H^{1}\left(\mathbb{C}^{m}, \mathcal{H}(T)_{\mathbb{C}^{m}}\right)=0$.

So we have $\pi^{*} X \cong \mathbb{C}^{m} \times T$ and by taking the universal covering $\mathbb{C}^{m} \times \mathbb{C}^{d} \rightarrow$ $\pi^{*} X \cong \mathbb{C}^{m} \times T$ and the composition with the covering $\pi^{\prime}: \pi^{*} X \rightarrow X$, we obtain the desired assertion.

Q.E.D.

Let us briefly recall again the standard way to look at the family $\mathcal{T}_{m}$ of complex tori of complex dimension $=m$. We fix a lattice $\Gamma$ of $\operatorname{rank} 2 m$, and we look at the complex ( $m$-dimensional) subspaces $V \subset \Gamma \otimes \mathbb{C}$ such that $V \oplus \bar{V}=\Gamma \otimes \mathbb{C}$ : to $V$ corresponds the complex torus $Y_{V}:=\Gamma \otimes \mathbb{C} /(\Gamma \oplus \bar{V})$. We finallyselect one of the two resulting connected components by requiring that the complex orientation of $V$ induces on $\Gamma \cong p_{V}(\Gamma)$ a standard orientation.

We define similarly $T_{U}:=\Lambda \otimes \mathbb{C} /(\Lambda \oplus \bar{U})$.

Consider now our principal holomorphic torus bundle $f: X \rightarrow Y$ over a complex torus $Y_{V}$ of dimension $m$, and with fibre a complex torus $T_{U}$ of dimension $d$ and let $\epsilon \in H^{2}(Y, \Lambda)=H^{2}(\Gamma, \Lambda)$ be the cohomology class classifying the central extension

$$
1 \rightarrow \Lambda \rightarrow \Pi \rightarrow \Gamma \rightarrow 1
$$

Lemma 2.3. It is possible to "tensor" the above exact sequence with $\mathbb{R}$, obtaining an exact sequence of Lie Groups

$$
1 \rightarrow \Lambda \otimes \mathbb{R} \rightarrow \Pi \otimes \mathbb{R} \rightarrow \Gamma \otimes \mathbb{R} \rightarrow 1
$$

such that $\Pi$ is a discrete subgroup of $\Pi \otimes \mathbb{R}$ and such that $X$ is diffeomorphic to the quotient

$$
M:=\Pi \otimes \mathbb{R} / \Pi
$$

Proof. Consider, as usual, the map

$$
\begin{gathered}
A: \Gamma \times \Gamma \rightarrow \Lambda, \\
A\left(\gamma, \gamma^{\prime}\right)=\left[\hat{\gamma}, \hat{\gamma^{\prime}}\right]=\hat{\gamma} \hat{\gamma}^{\prime}(\hat{\gamma})^{-1}\left(\hat{\gamma}^{\prime}\right)^{-1},
\end{gathered}
$$

where $\hat{\gamma}$ and $\hat{\gamma}^{\prime}$ are respective liftings to $\Pi$ of elements $\gamma, \gamma^{\prime} \in \Gamma$. We observe that since the extension (11) is central, the definition of $A$ does not depend on the choice of the liftings of $\gamma$, resp. $\gamma^{\prime}$ to $\Pi$.

As well known, $A$ is bilinear and alternating, so $A$ yields a cocycle in $H^{2}(\Gamma, \Lambda)$ which "classifies" the central extension (11). Let us review how does this more precisely hold. 
Assume that $\left\{\gamma_{1}, \ldots, \gamma_{2 m}\right\}$ is a basis of $\Gamma$ and choose fixed liftings $\hat{\gamma}_{i}$ of $\gamma_{i}$ in $\Pi$, for each $i=1, \ldots, 2 \mathrm{~m}$. Then automatically we have determined a canonical way to lift elements $\gamma \in \Gamma$ through:

$$
\gamma=\gamma_{1}^{n_{1}} \ldots \gamma_{2 m}^{n_{2 m}} \mapsto \hat{\gamma}:=\hat{\gamma}_{1}^{n_{1}} \ldots \hat{\gamma}_{2 m}^{n_{2 m}}
$$

Hence a canonical way to write the elements of $\Pi$ as products $\lambda \hat{\gamma}$, where $\lambda \in \Lambda$ and $\hat{\gamma}$ is as above.

Since $\forall i, j$, one has

$$
\hat{\gamma}_{i} \hat{\gamma}_{j}=A\left(\gamma_{i}, \gamma_{j}\right) \hat{\gamma}_{j} \hat{\gamma}_{i}
$$

we have a standard way of computing the products $(\lambda \hat{\gamma})\left(\lambda^{\prime} \hat{\gamma}^{\prime}\right)$ as $\lambda^{\prime \prime}\left(\widehat{\left(\gamma \gamma^{\prime}\right)}\right)$ in $\Pi$, where $\lambda^{\prime \prime}$ will be computed using $A$.

We can also view $\Pi$ as a group of affine transformations of $(\Lambda \otimes \mathbb{R}) \oplus(\Gamma \otimes \mathbb{R})$. In fact, $(\Lambda \otimes \mathbb{R}) \oplus(\Gamma \otimes \mathbb{R})$ is a real vector space with basis $\left\{\lambda_{1}, \ldots, \lambda_{2 d}, \gamma_{1}, \ldots, \gamma_{2 m}\right\}$ where $\left\{\lambda_{1}, \ldots, \lambda_{2 d}\right\}$ is a basis of $\Lambda$ and the action of $\Pi$ on $(\Lambda \otimes \mathbb{R}) \oplus(\Gamma \otimes \mathbb{R})$ is given as follows:

$\lambda_{i}$ acts on $(\Lambda \otimes \mathbb{R}) \oplus(\Gamma \otimes \mathbb{R})$ sending $(y, x)$ to $\left(y+\lambda_{i}, x\right)$, while the action of $\hat{\gamma}_{j}$ is defined using the multiplication $(\lambda \hat{\gamma}) \mapsto(\lambda \hat{\gamma}) \hat{\gamma}_{j}$.

More precisely if $y \in \Lambda \otimes \mathbb{R}, x=\sum x_{j} \gamma_{j} \in \Gamma \otimes \mathbb{R}, \gamma^{\prime}=\sum \nu_{h} \gamma_{h} \in \Gamma$, we have

$$
(y, x) \hat{\gamma}^{\prime}:=\left(y+\phi_{\gamma^{\prime}}(x), x+\gamma^{\prime}\right),
$$

where

$$
\phi_{\gamma^{\prime}}(x)=\sum_{j \geq h} x_{j} \nu_{h} A\left(\gamma_{j}, \gamma_{h}\right)=\sum_{j \geq h} x_{j} A_{j h} \nu_{h}={ }^{t} x T^{-} \gamma^{\prime},
$$

where $T^{-}$is the lower triangular part of the matrix $A$, so that we can write $A=T^{-}{ }^{t} T^{-}$.

Therefore we can endow $(\Lambda \otimes \mathbb{R}) \oplus(\Gamma \otimes \mathbb{R})=: \Pi \otimes \mathbb{R}$ with a Lie group structure defined by

$$
(y, x)\left(y^{\prime}, x^{\prime}\right)=\left(y+y^{\prime}+T^{-}\left(x, x^{\prime}\right), x+x^{\prime}\right),
$$

and the quotient $(\Pi \otimes \mathbb{R}) / \Pi$ of this Lie group by the discrete subgroup $\Pi$ is easily seen to be diffeomorphic to $X$.

Q.E.D.

Remark 2.4. We can change coordinates in $(\Lambda \otimes \mathbb{R}) \oplus(\Gamma \otimes \mathbb{R})$ in such a way that the action of the set $\hat{\Gamma} \cong \Gamma \subset \Gamma \otimes \mathbb{R}$ on $\Pi \otimes \mathbb{R}$ is given by

$$
(y, x) \hat{\gamma}=(y+A(x, \gamma)+2 S(\gamma, \gamma), x+\gamma),
$$

where $S\left(\gamma, \gamma^{\prime}\right)$ is a symmetric bilinear $\left(\frac{1}{4} \Lambda\right)$ - valued form, and $2 S(\gamma, \gamma) \in \Lambda$.

Proof.

Let us define the symmetric form $S:=-\frac{T^{-}+{ }^{t} T^{-}}{4}$, so that $T^{-}+2 S=$ $\frac{T^{-}-{ }^{t} T^{-}}{2}=\frac{A}{2}$.

Consider the map $F:(\Lambda \otimes \mathbb{R}) \oplus(\Gamma \otimes \mathbb{R}) \rightarrow(\Lambda \otimes \mathbb{R}) \oplus(\Gamma \otimes \mathbb{R})$ defined by $F(y, x)=(2(y+S(x, x)), x)=:(\eta, x)$. 
Then $\forall \hat{\gamma} \in \hat{\Gamma}$ we have an induced action

$$
\begin{gathered}
(\eta, x) \hat{\gamma}=F((y, x) \hat{\gamma})=F\left(y+T^{-}(x, \gamma), x+\gamma\right)= \\
\left(2 y+2 T^{-}(x, \gamma)+2 S(x+\gamma, x+\gamma), x+\gamma\right)=(\eta+A(x, \gamma)+2 S(\gamma, \gamma), x+\gamma),
\end{gathered}
$$

and we conclude observing that $2 S(\gamma, \gamma)=T^{-}(\gamma, \gamma) \in \Lambda$.

Q.E.D.

We recall from [Cat04] the First Riemann bilinear Relation: it is derived from the exact cohomology sequence

$H^{1}\left(Y, \mathcal{O}_{Y} \otimes U\right) \cong H^{1}\left(Y, \mathcal{H}(U)_{Y}\right) \rightarrow H^{1}\left(\mathcal{H}(T)_{Y}\right) \rightarrow^{c} \rightarrow H^{2}(Y, \Lambda) \rightarrow H^{2}\left(Y, \mathcal{H}(U)_{Y}\right)$

and says that the class $\epsilon$ maps to zero in $H^{2}\left(Y, \mathcal{H}(U)_{Y}\right)$. More concretely we have the

First Riemann Relation for principal holomorphic Torus Bundles

Let $A: \Gamma \times \Gamma \rightarrow \Lambda$ be the alternating bilinear map representing the cohomology class $\epsilon$ : then

$A \in \Lambda^{2}(\Gamma \otimes \mathbb{R})^{\vee} \otimes(\Lambda \otimes \mathbb{R}) \subset \Lambda^{2}(\Gamma \otimes \mathbb{C})^{\vee} \otimes(\Lambda \otimes \mathbb{C}) \subset \Lambda^{2}(V \oplus \bar{V})^{\vee} \otimes(U \oplus \bar{U})$, satisfies the property that its component in $\Lambda^{2}(\bar{V})^{\vee} \otimes(U)$ is zero.

We want now to explain in detail the bilinear algebra underlying the Riemann bilinear relation.

We observe preliminarly that one has a natural isomorphism $\Lambda^{2}(V \oplus \bar{V})^{\vee} \cong$ $\Lambda^{2}(V)^{\vee} \oplus\left(V^{\vee} \otimes \bar{V}\right)^{\vee} \oplus \Lambda^{2}(\bar{V})^{\vee}$, where the middle summand embeds by the wedge product : $w^{\prime} \otimes \bar{w} \mapsto w^{\prime} \wedge \bar{w}=w^{\prime} \otimes \bar{w}-\bar{w} \otimes w^{\prime}$.

Consider the bilinear form

$$
A \in \Lambda^{2}(\Gamma \otimes \mathbb{C})^{\vee} \otimes(\Lambda \otimes \mathbb{C})=\Lambda^{2}(V \oplus \bar{V})^{\vee} \otimes(U \oplus \bar{U}),
$$

satisfying the first bilinear relation and let us write

$$
A=B+\bar{B}
$$

where $B \in \Lambda^{2}(\Gamma \otimes \mathbb{C})^{\vee} \otimes U$, and $\bar{B} \in \Lambda^{2}(\Gamma \otimes \mathbb{C})^{\vee} \otimes \bar{U}$.

By the first bilinear relation $B=B^{\prime}+B^{\prime \prime}$, with $B^{\prime} \in \Lambda^{2}(V)^{\vee} \otimes U, B^{\prime \prime} \in$ $\left(V^{\vee} \otimes \bar{V}\right)^{\vee} \otimes U$.

Concretely, $A=B^{\prime}+B^{\prime \prime}+\overline{B^{\prime}}+\overline{B^{\prime \prime}}$, where $B^{\prime}$ is an alternating complex bilinear form. The fact that $B^{\prime \prime}$ is alternating reads out as:

$$
B^{\prime \prime}\left(v^{\prime}, \bar{v}\right)=-B^{\prime \prime}\left(\bar{v}, v^{\prime}\right) \forall v, v^{\prime} \in V
$$

whereas conjugation of tensors reads out as:

$$
\bar{B}(\bar{x}, \bar{y})=\overline{B(x, y)} \forall x, y \Rightarrow \overline{B^{\prime \prime}}\left(\bar{v}, v^{\prime}\right)=\overline{B^{\prime \prime}\left(v, \bar{v}^{\prime}\right)} .
$$

Definition 2.5. We define the associated (vector-valued) Hermitian bilinear form

$$
D: V \times V \rightarrow \Lambda \otimes \mathbb{C}
$$

through

$$
D\left(v_{1}, v_{2}\right):=i A\left(v_{1}, \overline{v_{2}}\right) .
$$


In fact $D$ is clearly complex linear in $v_{1}$ and complex antilinear in $v_{2}$, and $D\left(v_{2}, v_{1}\right):=i A\left(v_{2}, \bar{v}_{1}\right)=-i A\left(\overline{v_{1}}, v_{2}\right)=-i \overline{A\left(v_{1}, \bar{v}_{2}\right)}=\overline{D\left(v_{1}, v_{2}\right)}$.

We have

$D\left(v_{1}, v_{2}\right):=i A\left(v_{1}, \overline{v_{2}}\right)=i\left(B^{\prime \prime}\left(v_{1}, \overline{v_{2}}\right)+\overline{B^{\prime \prime}}\left(v_{1}, \overline{v_{2}}\right)\right)=i B^{\prime \prime}\left(v_{1}, \overline{v_{2}}\right)+\overline{i B^{\prime \prime}\left(v_{2}, \overline{v_{1}}\right)}$.

Remark 2.6. One can view $D(v, v): V \rightarrow \Lambda \otimes \mathbb{R}$ as a real linear system (of dimension $\leq 2 d$ ) of Hermitian forms on $V$, and its discriminant

$\Delta_{D}=\left\{\left(\lambda_{i}\right) \mid \operatorname{det}\left(\sum_{1}^{2 d} \lambda_{i} D_{i}\right)=0\right\}$ will be a real hypersurface of degree $m$ in the projective space $\mathbb{P}\left((\Lambda \otimes \mathbb{C})^{\vee}\right)$.

Observe that this linear system is independent of the choice of $U$, but it depends upon the choice of $V$.

The geometry of $\Delta_{D}$ and more generally the geometry of the linear rational map $\mathbb{P}\left((\Lambda \otimes \mathbb{R})^{\vee}\right) \rightarrow \mathbb{P}($ Herm $(m, \mathbb{C}))$ produces holomorphic invariants of the complex structure.

These are however related to the topological invariants given by the linear rational map $\left.\mathbb{P}\left(\mathbb{R}^{2 d}\right) \cong \mathbb{P}\left((\Lambda \otimes \mathbb{R})^{\vee}\right) \rightarrow \mathbb{P}\left(\Lambda^{2}(\Gamma \otimes \mathbb{R})^{\vee}\right)\right) \cong \mathbb{P}\left(\Lambda^{2}\left(\mathbb{R}^{2 m}\right)\right)$.

\section{Appell Humbert families and Kodaira Spencer map}

We shall consider in this section the family given by the pairs of subspaces satisfying the Riemann bilinear relations, and the main goal will be to compute explicitly its tangent space and its Kodaira Spencer map.

Definition 3.1. Given $A$ as above, we define $\mathcal{T B}_{A}$ as the subset of the product of Grassmann Manifolds $\operatorname{Gr}(m, 2 m) \times G r(d, 2 d)$ defined by

$$
\begin{gathered}
\mathcal{T B}_{A}=\{(V, U) \in G r(m, 2 m) \times G r(d, 2 d) \mid V \cap \bar{V}=(0), \\
\left.U \cap \bar{U}=(0), \mid \text { the component of } A \text { in } \Lambda^{2}(\bar{V})^{\vee} \otimes U \text { is }=0\right\} .
\end{gathered}
$$

This complex space is called the Appell Humbert space of Torus Bundles.

Since this space is not connected, we restrict ourselves to its intersection with $\mathcal{T}_{m} \times \mathcal{T}_{d}$, i.e., we fix respective complex structures which have the same orientation as fixed orientations of $\Lambda$, resp. $\Gamma$.

One sees immediately that $\mathcal{T} \mathcal{B}_{A}$ is a complex analytic variety of codimension at most $d m(m-1) / 2$.

Note however that, for $d \geq 3, m>>0$ we get a negative expected dimension. The structure of these complex spaces has to be investigated in general, for our present purposes we limit ourselves to establish the following

Lemma 3.2. If $d=1$ then the open set $\mathcal{T} \mathcal{B}_{A} \cap\left(\mathcal{T}_{m} \times \mathcal{T}_{d}\right)$ is connected.

Proof.

Since $\mathcal{T} \mathcal{B}_{A} \cap\left(\mathcal{T}_{m} \times \mathcal{T}_{1}\right)$ fibres onto the upper half plane $\mathcal{T}_{1}$, which is connected, it suffices to show that each fibre is connected.

Our method of proof will be quite simple minded. We shall consider the fibration onto $\mathcal{T}_{1}$, but without restricting to $\mathcal{T}_{m}$, and considering instead the 
larger open set $\{V \mid V \cap \bar{V}=(0)\}$ (which has two connected components) and will show that the corresponding fibre has exactly two connected components.

Observe then that, if we fix $U$ and write $\Lambda \otimes \mathbb{C}=U \oplus \bar{U}$, the connectivity of our space in independent of the given integral structures, and it amounts to the following problem: we are given an oriented real vector space $W(W=\Gamma \otimes \mathbb{R})$ and a real alternating map $A: \Lambda^{2}(W) \rightarrow U \oplus \bar{U}$, so that clearly we can write $A=B \oplus \bar{B}$.

We are seeking for the subspaces $V$ such that $\bar{V}$ is isotropic for $B$, such that $W \otimes \mathbb{C}=V \oplus \bar{V}$, and such that the complex orientation of $V$ induces the fixed orientation on $W$.

Since the complex linear group is connected, it is sufficient to show the connectedness of the variety of frames $v_{1}, \ldots v_{m}$ for $\bar{V}$.

There are two conditions to be satisfied in the choice of $v_{1}, \ldots v_{m}$ :

1) the closed condition that $v_{i}$ belongs to the complex subspace

$$
W_{i}:=\left\{v \mid B\left(v, v_{j}\right)=0 \forall 1 \leq j \leq i-1\right\},
$$

which has obviously complex dimension at least $2 m-i+1$

2) the open condition that $v_{i}$ belongs to the open set

$\Omega_{i}:=\left\{v_{i} \mid v_{1}, \overline{v_{1}}, \ldots v_{i}, \overline{v_{i}}\right.$ are $\mathbb{C}$ - linearly independent $\}$.

We show the connectedness of the variety $\mathcal{F}_{i}$ of partial frames $v_{1}, \ldots v_{i}$ satisfying 1) and 2) by induction on $i$.

For $i=1$, we consider an arbitrary vector $v_{1}$, belonging to the open set $\Omega_{1}:=\left\{v_{1} \mid v_{1}, \overline{v_{1}}\right.$ are $\mathbb{C}$ - linearly independent $\}$.

Observe that the complement of $\Omega_{1}$ has real codimension $2 m-1$, thus it does not disconnect a vector space of real dimension $4 m$, as soon as $m \geq 2$.

A similar argument applied to the projection of $v_{i}$ into the quotient vector space $\tilde{W}_{i}:=W \otimes \mathbb{C} /<<v_{1}, \overline{v_{1}}, \ldots v_{i-1}, \overline{v_{i-1}}>>$, which has complex dimension $2 m-2 i+2$, shows that $\Omega_{i}$ has a complementary set of real codimension $2 m-2 i+1$, thus we conclude that $\Omega_{i} \cap W_{i}$ is connected for $i<m$.

In the final step instead, we have to remove the zero locus of the Hermitian form (of $v_{m}$ )

$$
\operatorname{det}\left(v_{1}, \overline{v_{1}}, \ldots v_{m}, \overline{v_{m}}\right)=0
$$

We conclude by observing that a Hermitian form has even positivity and even negativity, thus the complementary set of its zero locus has exactly two connected components.

Therefore we get, by induction on $i$, that the variety $\mathcal{F}_{i}$ of partial frames $v_{1}, \ldots v_{i}$ satisfying 1 ) and 2) is connected for $i \leq m-1$, while the variety $\mathcal{F}_{m}$ of such frames has at most two connected components for $i=m$.

$$
\text { Q.E.D. }
$$

Definition 3.3. The standard (Appell-Humbert) family of torus bundles parametrized by $\mathcal{T B}_{A}$ is the family of principal holomorphic torus bundles $X_{V, U}$ over $Y:=Y_{V}$ and with fibre $T:=T_{U}$ determined by the cocycle in 
$H^{1}\left(Y, \mathcal{H}(T)_{Y}\right)$ obtained by taking $f_{\gamma}(v)$ which is the class modulo $\Lambda$ of

$$
F_{\gamma}(v):=B^{\prime}\left(v, p_{V}(\gamma)\right)+2 B^{\prime \prime}\left(v, \overline{p_{V}(\gamma)}\right)+B^{\prime \prime}\left(p_{V}(\gamma), \overline{p_{V}(\gamma)}\right), \forall v \in V
$$

In other words, $X_{V, U}$ is the quotient of $T_{U} \times V$ by the action of $\Gamma$ such that $\gamma([u], v)=\left(\left[u+B^{\prime}\left(v, p_{V}(\gamma)\right)+2 B^{\prime \prime}\left(v, \overline{p_{V}(\gamma)}\right)+B^{\prime \prime}\left(p_{V}(\gamma), \overline{p_{V}(\gamma)}\right)\right], v+p_{V}(\gamma)\right)$.

Remark 3.4. The above formula differs from the formula given in Definition 6.4 of [Cat04], where an identification of $\Gamma \otimes \mathbb{R}$ with $V$ was used, and thus $A(z, \gamma)$ was identified with $B(z, \gamma)$. In the latter formula one had thus

$$
\begin{aligned}
& -B^{\prime}(v, \gamma)-B^{\prime \prime}\left(v, \overline{p_{V}(\gamma)}\right) \text { instead of } \\
& B^{\prime}(v, \gamma)+2 B^{\prime \prime}\left(v, \overline{p_{V}(\gamma)}\right)+B^{\prime \prime}\left(p_{V}(\gamma), \overline{p_{V}(\gamma)}\right) .
\end{aligned}
$$

However, this alteration does not affect the proofs of Theorems 6.10, 6.11, 6.12 since in those proofs $B^{\prime}$ and $B^{\prime \prime}$ were playing separate roles, and multiplication by 2 or -1 does not alter the condition that a certain expression be zero.

We also recall from Cat04 the definition of the complete Appell-Humbert space.

Definition 3.5. Given $A$ as above we define

$$
\mathcal{T}^{\prime} \mathcal{B}_{A}=\left\{(V, U, \phi) \mid(V, U) \in \mathcal{T} \mathcal{B}_{A}, \phi \in H^{1}\left(Y_{V}, \mathcal{H}(U)_{Y_{V}}\right) \cong \bar{V}^{\vee} \otimes U\right\} .
$$

The complete Appell-Humbert family of torus bundles parametrized by $\mathcal{T}^{\prime} \mathcal{B}_{A}$ is the family of principal holomorphic torus bundles $X_{V, U, \phi}$ on $Y:=Y_{V}$ and with fibre $T:=T_{U}$ determined by the cocycle in $H^{1}\left(Y, \mathcal{H}(T)_{Y}\right)$ obtained by taking the sum of $f_{\gamma}(z)$ with the cocycle $\phi \in H^{1}\left(Y_{V}, \mathcal{H}(U)_{Y_{V}}\right) \cong H^{1}\left(Y, \mathcal{O}_{Y}^{d}\right)$.

Finally we have the following

Theorem 3.6. Cat04

Any principal holomorphic torus bundle with extension class isomorphic to $\epsilon \in H^{2}(\Gamma, \Lambda)$ occurs in the complete Appell-Humbert family $\mathcal{T}^{\prime} \mathcal{B}_{A}$.

Without reproving the above theorem, we want to give a differential geometric explanation of the cocycle formula above.

The idea is very simple : we consider the homogeneous space $M:=\Pi \otimes \mathbb{R} / \Pi$ as a fixed differentiable manifold, and we use the canonical identification of the tangent space at the identity in $\Pi \otimes \mathbb{R}$ with $(\Lambda \otimes \mathbb{R}) \oplus(\Gamma \otimes \mathbb{R})$ to define a right invariant almost complex structure on $\Pi \otimes \mathbb{R}$ by translating $U \oplus V$.

This induces a right invariant almost complex structure on $M$, and we prove that if the Riemann bilinear relations hold, then the complex structure is integrable. Observe that in general $\Pi \otimes \mathbb{R}$ will not have a complex Lie group structure, because, even if right multiplication is holomorphic, the inverse mapping needs not be holomorphic.

This interpretation will be quite useful in order to understand the Kodaira Spencer map of the Appell Humbert families. 
Proposition 3.7. Let us consider the unique subbundle $T^{(1,0)} \subset T_{M} \otimes \mathbb{C}$ of the complexified tangent bundle of $M:=\Pi \otimes \mathbb{R} / \Pi$, which is invariant by right translations, and is such that under the identification of the tangent space at the identity in $\Pi \otimes \mathbb{R}$ with $(\Lambda \otimes \mathbb{R}) \oplus(\Gamma \otimes \mathbb{R})$, we have $T_{I d}^{(1,0)}=U \oplus V$.

Then, using the other identification of $T_{M} \otimes \mathbb{C}$ with the trivial bundle $(\Lambda \otimes$ $\mathbb{C}) \oplus(\Gamma \otimes \mathbb{C})$, provided by the diffeomorphism of $\Pi \otimes \mathbb{R}$ with $(\Lambda \otimes \mathbb{R}) \oplus(\Gamma \otimes \mathbb{R})$, with coordinates $(y, x)$, then

$$
\begin{gathered}
T_{(y, x)}^{(1,0)}=\{(u+A(v, x), v) \mid u \in U, v \in V\}= \\
=\left\{u+v+\overline{B^{\prime \prime}}\left(v, \overline{p_{V}(x)}\right) \mid u \in U, v \in V\right\} \subset U \oplus V \oplus \bar{U}
\end{gathered}
$$

Proof. $\forall g=(y, x) \in \Pi \otimes \mathbb{R}$, since

$$
\left(y^{\prime}, x^{\prime}\right)(y, x)=\left(y^{\prime}+y+A\left(x^{\prime}, x\right)+2 S(x, x), x^{\prime}+x\right),
$$

we have

$$
T_{g}^{(1,0)}=\left\{R_{g_{*}}(u, v) \mid u \in U, v \in V\right\}=\{(u+A(v, x), v) \mid u \in U, v \in V\}
$$

which equals, as claimed,

$$
\begin{gathered}
=\left\{\left(u+B^{\prime}\left(v, p_{V}(x)\right)+B^{\prime \prime}\left(v, \overline{p_{V}(x)}\right)+\overline{B^{\prime \prime}}\left(v, \overline{p_{V}(x)}\right), v\right)=\right. \\
\left.=\left(u^{\prime}+\overline{B^{\prime \prime}}\left(v, \overline{p_{V}(x)}\right), v\right) \mid u^{\prime} \in U, v \in V\right\} .
\end{gathered}
$$

Q.E.D.

It is now clear that this subbundle $T^{(1,0)}$ is stable under the operation of the discrete group $\Pi$ given by multiplication from the right.

Remark 3.8. We observe that if we denote by $i: \Pi \otimes \mathbb{R} \rightarrow \Pi \otimes \mathbb{R}$ the inverse map sending $g$ to $g^{-1}$ and by $D(i)_{g}$ the differential of $i$ at the point $g \in \Pi \otimes \mathbb{R}$, we have in general that $D(i)_{g}\left(T_{g}^{(1,0)}\right) \neq T_{g^{-1}}^{(1,0)}$.

Proof. If $g=(y, x) \in \Pi \otimes \mathbb{R}, g^{-1}=i(y, x)=(-y-2 S(x, x),-x)$, so we have

$$
D(i)_{g}(u+A(v, x), v)=(-u-A(v, x)-4 S(x, v),-v) .
$$

On the other hand

$$
\begin{aligned}
& T_{g^{-1}}^{(1,0)}=\{(u+A(v,-x), v) \mid u \in U, v \in V\} \\
\neq & \{(u+A(v, x)+4 S(x, v), v) \mid u \in U, v \in V\} .
\end{aligned}
$$

Q.E.D.

Lemma 3.9. The above almost complex structure on the differentiable manifold $M:=\Pi \otimes \mathbb{R} / \Pi$ that we have defined is integrable iff the Riemann bilinear relations are satisfied for the pair $(U, V)$. 
Proof.

We have already observed the necessity of the Riemann bilinear relations, so let us just prove the "if" part.

We have $\forall g=(y, x) \in \Pi \otimes \mathbb{R}$, the holomorphic tangent subbundle

$$
T_{g}^{(1,0)}=\left\{u+v+\overline{B^{\prime \prime}}\left(v, \overline{p_{V}(x)}\right) \mid u \in U, v \in V\right\} \subset U \oplus V \oplus \bar{U}
$$

and its conjugate

$$
T_{g}^{(0,1)}=\overline{T_{g}^{(1,0)}}=\left\{\bar{u}+\bar{v}+B^{\prime \prime}\left(\bar{v}, p_{V}(x)\right) \mid \bar{u} \in \bar{U}, \bar{v} \in \bar{V}\right\} \subset \bar{U} \oplus \bar{V} \oplus U .
$$

We have to show that the holomorphic cotangent bundle, which is the annihilator of $T_{g}^{(0,1)}$, is generated by the differentials of certain local functions. But we are lucky and we can indeed produce these functions globally.

Consider in fact the diffeomorphism $\Psi: \Pi \otimes \mathbb{R} \rightarrow U \oplus V$ given by $(y, x) \mapsto\left(p_{U}(y)-B^{\prime \prime}\left(x, p_{V}(x)\right), p_{V}(x)\right)=\left(p_{U}(y)-B^{\prime \prime}\left(\overline{p_{V}(x)}, p_{V}(x)\right), p_{V}(x)\right)$.

$\Psi$ is a diffeomorphism since one can in fact compute the inverse map explicitly as

$$
(u, v) \mapsto\left(u+\bar{u}+B^{\prime \prime}(\bar{v}, v)+\overline{B^{\prime \prime}(\bar{v}, v)}, v+\bar{v}\right)
$$

It is easy to verify that the differentials of the coordinates of $\Psi$ annihilate $T_{g}^{(0,1)}$.

$$
\text { Q.E.D. }
$$

Corollary 3.10. Given $U, V$ satisying the Riemann bilinear relations, the corresponding integrable complex structure induces a holomorphic action of $\Pi$ on $U \oplus V$ which corresponds to the cocycle given in definition 3.3 .

Proof. We use the diffeomorphism $\Psi$ used previously to induce an action of $\Pi$ on $U \oplus V$. This action is holomorphic since the complex structure is invariant by right translation.

We just have to read out $\Psi \circ R_{g} \circ \Psi^{-1}(u, v)$ using the shorthand notation $g=(y, x)$ and $p_{U}(y):=y_{U}, p_{V}(x):=x_{V}$ (thus, for instance $\left.x=x_{V}+\overline{x_{V}}\right)$. We have:

$$
\begin{gathered}
\Psi \circ R_{g} \circ \Psi^{-1}(u, v)=\Psi \circ R_{g}\left(u+\bar{u}+B^{\prime \prime}(\bar{v}, v)+\overline{B^{\prime \prime}(\bar{v}, v)}, v+\bar{v}\right)= \\
=\Psi\left(y+u+\bar{u}+B^{\prime \prime}(\bar{v}, v)+\overline{B^{\prime \prime}(\bar{v}, v)}+A(v+\bar{v}, x), x+v+\bar{v}\right)= \\
=\left(y_{U}+u+B^{\prime \prime}(\bar{v}, v)+B(v+\bar{v}, x)-B^{\prime \prime}\left(x+v+\bar{v}, x_{V}+v\right), x_{V}+v\right)= \\
=\left(y_{U}+u+B^{\prime}\left(v, x_{V}\right)+2 B^{\prime \prime}\left(v, \overline{x_{V}}\right)+B^{\prime \prime}\left(x_{V}, \overline{x_{V}}\right), x_{V}+v\right) .
\end{gathered}
$$

Q.E.D.

We give now a description of the tangent space to $\mathcal{T} \mathcal{B}_{A}$, observing that $\mathcal{T}^{\prime} \mathcal{B}_{A}=\left\{(V, U, \phi) \mid(V, U) \in \mathcal{T} \mathcal{B}_{A}, \phi \in \bar{V}^{\vee} \otimes U\right\}$ is the restriction to $\mathcal{T} \mathcal{B}_{A}$ of a vector bundle on $G(m, 2 m) \times G(d, 2 d)$.

For $(V, U) \in \mathcal{T B}_{A}$, the tangent space to $\mathcal{T} \mathcal{B}_{A}$ in $(V, U)$ is a subspace of the tangent space to the product of the Grassmannians,

$$
T_{\mathcal{T B}_{A},(V, U)} \subset T_{G r(m, 2 m) \times G r(d, 2 d),(V, U)} \cong \operatorname{Hom}(V, \bar{V}) \oplus \operatorname{Hom}(U, \bar{U}) .
$$


Let $(L, M) \in \operatorname{Hom}(V, \bar{V}) \oplus \operatorname{Hom}(U, \bar{U})$ be a tangent vector. In order to determine the Zariski tangent space to $\mathcal{T} \mathcal{B}_{A}$, we work over the ring of dual numbers $\mathbb{C}[\epsilon] /\left(\epsilon^{2}\right)$ and impose the Riemann bilinear conditions infinitesimally, i.e., considering the infinitesimal variations of $V$, resp. $U$, given by the graph $V_{\epsilon}$ of $\epsilon L: V \oplus \epsilon V \rightarrow \bar{V} \oplus \epsilon \bar{V}$, resp. the graph $U_{\epsilon}$ of $\epsilon M$. Concretely, $V_{\epsilon}=$ $\left\{v+\epsilon v^{\prime}+\epsilon L v \mid v, v^{\prime} \in V\right\}$, and we want $A\left(V_{\epsilon}, V_{\epsilon}\right) \subset U_{\epsilon}$.

$$
A\left(v+\epsilon v_{2}+\epsilon L(v), v^{\prime}+\epsilon v_{2}^{\prime}+\epsilon L\left(v^{\prime}\right)\right) \in U_{\epsilon}, \forall v, v^{\prime}, v_{2}, v_{2}^{\prime} \in V .
$$

So we must have elements $u_{0}, u_{0}^{\prime} \in U$ such that

$$
A\left(v+\epsilon v_{2}+\epsilon L(v), v^{\prime}+\epsilon v_{2}^{\prime}+\epsilon L\left(v^{\prime}\right)\right)=u_{0}+\epsilon u_{0}^{\prime}+\epsilon M u_{0},
$$

i.e.,

- $A\left(v, v^{\prime}\right)=u_{0}$

- $A\left(L v, v^{\prime}\right)+A\left(v, L v^{\prime}\right)+A\left(v, v_{2}^{\prime}\right)+A\left(v_{2}, v^{\prime}\right)=u_{0}^{\prime}+M u_{0}$.

Since $(V, U) \in \mathcal{T B}_{A}, A\left(v, v^{\prime}\right)=B\left(v, v^{\prime}\right)=B^{\prime}\left(v, v^{\prime}\right)$, and the second equation may be rewritten as:

$B^{\prime}\left(v, v_{2}^{\prime}\right)+B^{\prime}\left(v_{2}, v^{\prime}\right)-B^{\prime \prime}\left(v^{\prime}, L v\right)+\overline{B^{\prime \prime}\left(\overline{L v}, \overline{v^{\prime}}\right)}+B^{\prime \prime}\left(v, L v^{\prime}\right)-\overline{B^{\prime \prime}\left(\overline{L v^{\prime}}, \bar{v}\right)}=u_{0}^{\prime}+M u_{0}$.

Finally, observing that $u_{0}^{\prime} \in U, M u_{0} \in \bar{U}$, we must have

$$
\begin{gathered}
u_{0}^{\prime}=B^{\prime}\left(v, v_{2}^{\prime}\right)+B^{\prime}\left(v_{2}, v^{\prime}\right)-B^{\prime \prime}\left(v^{\prime}, L v\right)+B^{\prime \prime}\left(v, L v^{\prime}\right), \\
M u_{0}=M B^{\prime}\left(v, v^{\prime}\right), \\
M B^{\prime}\left(v, v^{\prime}\right)=\overline{B^{\prime \prime}\left(\overline{L v}, \overline{v^{\prime}}\right)}-\overline{B^{\prime \prime}\left(\overline{L v^{\prime}}, \bar{v}\right)},
\end{gathered}
$$

and the last one is the equation defining the tangent space to $\mathcal{T} \mathcal{B}_{A}$ in $(V, U)$, which we rewrite using complex conjugation as

$$
\overline{M B^{\prime}\left(v, v^{\prime}\right)}=B^{\prime \prime}\left(\overline{L v}, \overline{v^{\prime}}\right)-B^{\prime \prime}\left(\overline{L v^{\prime}}, \bar{v}\right) .
$$

Proposition 3.11. Let $A: \Gamma \times \Gamma \rightarrow \Lambda$ be non zero.

If $m=2, d=1$, i.e., $\Gamma \cong \mathbb{Z}^{4}, \Lambda \cong \mathbb{Z}^{2}$, both Appell - Humbert spaces $\mathcal{T} \mathcal{B}_{A}$ and $\mathcal{T}^{\prime} \mathcal{B}_{A}$ are smooth.

Proof.

Recall once more that $\mathcal{T}^{\prime} \mathcal{B}_{A}=\left\{(V, U, \phi) \mid(V, U) \in \mathcal{T H}_{A}, \phi \in \bar{V}^{\vee} \otimes U\right\}$ is the restriction to $\mathcal{T} \mathcal{B}_{A}$ of a vector bundle on $G(m, 2 m) \times G(d, 2 d)$ (here $m=2$, $d=1$ ), so it suffices to prove that $\mathcal{T} \mathcal{B}_{A}$ is smooth.

We have shown that the tangent space to $\mathcal{T B}_{A}$ in $(V, U)$ is the subspace of $\operatorname{Hom}(V, \bar{V}) \oplus \operatorname{Hom}(U, \bar{U})$ given by kernel of the following linear map

$$
\begin{gathered}
G: \operatorname{Hom}(V, \bar{V}) \oplus \operatorname{Hom}(U, \bar{U}) \rightarrow \operatorname{Hom}\left(\Lambda^{2}(V), U\right), \\
(L, M) \mapsto\left[\left(v, v^{\prime}\right) \mapsto \overline{M B^{\prime}\left(v, v^{\prime}\right)}-B^{\prime \prime}\left(\overline{L v}, \overline{v^{\prime}}\right)+B^{\prime \prime}\left(\overline{L v^{\prime}}, \bar{v}\right)\right],
\end{gathered}
$$

thus it suffices to show that $G$ is surjective. Since $m=2$ and $d=1$, the dimension of the space $\operatorname{Hom}\left(\Lambda^{2}(V), U\right)$ is one, and we only have to prove that the map $G$ is non zero. 
For this purpose we observe that if $B^{\prime} \neq 0, G(0, M) \neq 0$, if $M \neq 0$.

If $B^{\prime}=0$, we have $B^{\prime \prime} \neq 0$ and there must exist $L \in H o m(V, \bar{V})$ such that $G(L, 0) \neq 0$.

In fact, otherwise we would have

$$
B^{\prime \prime}\left(\overline{L v}, \overline{v^{\prime}}\right)=B^{\prime \prime}\left(\overline{L v^{\prime}}, \bar{v}\right), \forall L \in \operatorname{Hom}(V, \bar{V}), \forall v, v^{\prime} \in V .
$$

For each $v, v^{\prime}, v^{\prime \prime} \in V$, with $v, v^{\prime}$ linearly independent, there is $L$ such that $L v=\bar{v}^{\prime \prime}=L v^{\prime}$, whence we get $B^{\prime \prime}\left(v^{\prime \prime}, \overline{v^{\prime}-v}\right)=0$ and it follows right away that $B^{\prime \prime}=0$.

Q.E.D.

Remark 3.12. The previous proposition contradicts remark 6.13 of Cat04, which however was due to a trivial misprint (exchanging $H^{1}\left(\mathcal{O}_{X}\right)$ and $\left.H^{1}\left(\mathcal{O}_{Y}\right)\right)$.

If $d=1$ and $m \geq 3$, however, $\mathcal{T} \mathcal{B}_{A}$ is singular at the points where $B^{\prime \prime}=0$.

\section{Proof.}

Let $(V, U) \in \mathcal{T} \mathcal{B}_{A}$.

Since $d=\operatorname{dim}(U)=1$, the map

$$
\begin{gathered}
G: \operatorname{Hom}(V, \bar{V}) \oplus \operatorname{Hom}(U, \bar{U}) \rightarrow \operatorname{Hom}\left(\Lambda^{2}(V), U\right), \\
(L, M) \mapsto\left[\left(v, v^{\prime}\right) \mapsto \overline{M B^{\prime}\left(v, v^{\prime}\right)}-B^{\prime \prime}\left(\overline{L v}, \overline{v^{\prime}}\right)+B^{\prime \prime}\left(\overline{L v^{\prime}}, \bar{v}\right)\right]
\end{gathered}
$$

has rank one if $B^{\prime \prime}=0$.

Q.E.D.

Now we want to determine the Kodaira Spencer map for the Appell-Humbert family.

Recall that $\forall g=(y, x) \in \Pi \otimes \mathbb{R}$, the holomorphic tangent subbundle is

$$
T_{g}^{(1,0)}=\left\{\left(u+\overline{B^{\prime \prime}}(v, x), v\right) \mid u \in U, v \in V\right\} \subset U \oplus V \oplus \bar{U}
$$

thus its conjugate is

$$
T_{g}^{(0,1)}=\overline{T_{g}^{(1,0)}}=\left\{\left(\bar{u}+B^{\prime \prime}(\bar{v}, x), \bar{v}\right) \mid \bar{u} \in \bar{U}, \bar{v} \in \bar{V}\right\} \subset \bar{U} \oplus \bar{V} \oplus U .
$$

Recall that one way to look at the Kodaira Spencer map is to regard it as associating to $\bar{M} \in \operatorname{Hom}(\bar{U}, U), \bar{L} \in \operatorname{Hom}(\bar{V}, V)$ a linear map $R=\rho(L, M) \in$ $\operatorname{Hom}\left(T_{g}^{(0,1)}, T_{g}^{(1,0)}\right)$ which gives the infinitesimal variation of the subspace $T_{g}^{(0,1)}$ as the graph of $I+\epsilon R$, where $I$ denotes the identity of $T_{g}^{(0,1)}$.

So, for $\bar{M} \in \operatorname{Hom}(\bar{U}, U), \bar{L} \in \operatorname{Hom}(\bar{V}, V)$, we consider the space

$$
\begin{aligned}
& \left(T_{g}^{(0,1)}\right)_{\epsilon}:=\left\{\left(\bar{u}+\epsilon \bar{M} \bar{u}+B^{\prime \prime}(\bar{v}+\epsilon \bar{L} \bar{v}, x), \bar{v}+\epsilon \bar{L} \bar{v}\right) \mid \bar{u} \in \bar{U}, \bar{v} \in \bar{V}\right\}= \\
& =\left\{\left(\bar{u}+B^{\prime \prime}(\bar{v}, x)+\epsilon\left(\bar{M} \bar{u}+B^{\prime \prime}(\bar{L} \bar{v}, x)\right), \bar{v}+\epsilon \bar{L} \bar{v}\right) \mid \bar{u} \in \bar{U}, \bar{v} \in \bar{V}\right\} .
\end{aligned}
$$

We obtain $R$ simply by projecting the variation (i.e., the terms divisible by $\epsilon)$ to $T_{g}^{(1,0)}$. 
We write this projection using the decomposition: $u+\bar{u}^{\prime}+v+\bar{v}^{\prime}=$ $=\left(u-B^{\prime \prime}\left(\overline{v^{\prime}}, x\right)\right)+\left(\overline{u^{\prime}}-\overline{B^{\prime \prime}}(v, x)\right)+\left(v+\overline{B^{\prime \prime}}(v, x)\right)+\left(\overline{v^{\prime}}+B^{\prime \prime}\left(\overline{v^{\prime}}, x\right)\right)$

So we have a map

$$
T_{g}^{(0,1)} \rightarrow T_{g}^{(1,0)},
$$

and using the isomorphism $U \oplus V \cong T_{g}^{(1,0)}$ provided by the choice of $g=$ $(y, x),(u, v) \mapsto u+v+\overline{B^{\prime \prime}}(v, x)$, we obtain

$$
\begin{gathered}
R: \bar{U} \oplus \bar{V} \rightarrow U \oplus V, \\
R:(\bar{u}, \bar{v}) \mapsto\left(\bar{M} \bar{u}+B^{\prime \prime}(\bar{L} \bar{v}, x), \bar{L} \bar{v}\right) .
\end{gathered}
$$

We can partly summarize the above description (dependent upon the local choice of a splitting ) as follows:

Lemma 3.13. Consider the exact sequences of vector bundles on $X$ :

$$
0 \rightarrow U \rightarrow T^{(1,0)} \rightarrow V \rightarrow 0, \quad 0 \rightarrow \bar{U} \rightarrow T^{(0,1)} \rightarrow \bar{V} \rightarrow 0 .
$$

Then if $R=\rho(L, M, \phi)$ is the Kodaira Spencer image of the tangent vector $(L, M, \phi)$ to the complete Appell Humbert space, then the image of $R$ in $\operatorname{Hom}\left(T^{(0,1)}, V\right)$ is the image of $\bar{L} \in \operatorname{Hom}(\bar{V}, V)$, and the image of $R$ in $\operatorname{Hom}\left(\bar{U}, T^{(1,0)}\right)$ is the image of $\bar{M} \in \operatorname{Hom}(\bar{U}, U)$.

\section{On the versality of the complete Appell Humbert family}

We recall for the reader's benefit some results from [Cat04:

Theorem 4.1. The cokernel of $0 \rightarrow H^{0}\left(\Omega_{Y}^{1}\right) \rightarrow H^{0}\left(\Omega_{X}^{1}\right)$ is the subspace of $U^{\vee}$ which annihilates the image of the Hermitian part of $A$, i.e., of the component $B^{\prime \prime}$ in $\left[(V \otimes \bar{V})^{\vee} \otimes(U)\right]$.

It follows in particular that $X$ is parallelizable if and only if the Hermitian part of $A$ is zero.

Corollary 4.2. The space $H^{0}\left(d \mathcal{O}_{X}\right)$ of closed holomorphic 1-forms on $X$ contains the pull- back of $H^{0}\left(\Omega_{Y}^{1}\right)$ with cokernel the subspace $U^{*}$ of $U^{\vee}$ which annihilates the image of $B$, i.e., $U^{*}=\{\beta \mid \beta \circ B(z, \gamma)=0, \forall \gamma, \forall z\}$.

Theorem 4.3. The cokernel of $0 \rightarrow H^{1}\left(\mathcal{O}_{Y}\right) \rightarrow H^{1}\left(\mathcal{O}_{X}\right)$ is the subspace of $\bar{U}^{\vee}$ which annihilates the image of the anti-complex component of $A$, i.e., of the conjugate of the component $B^{\prime}$ in $\left[\left(\Lambda^{2} V\right)^{\vee} \otimes(U)\right]$.

In order to analyse the problem of describing the small deformations of principal holomorphic torus bundles over tori we need to calculate the cohomology groups of the tangent sheaf $\Theta_{X}$.

These fit into the following exact sequences, according to

Corollary 4.4. Cat04 $H^{i}\left(\Theta_{X}\right)$ fits into a short exact sequence

$$
0 \rightarrow \operatorname{cokerb}_{i-1} \rightarrow H^{i}\left(\Theta_{X}\right) \rightarrow \text { kerb }_{i} \rightarrow 0,
$$

where $b_{i}: V \otimes H^{i}\left(\mathcal{O}_{X}\right) \rightarrow U \otimes H^{i+1}\left(\mathcal{O}_{X}\right)$ is given by cup product and contraction with $\left.B^{\prime \prime} \in[(\bar{V}) \otimes V)^{\vee} \otimes(U)\right]$. 
We want to give now a more explicit description of these groups for $i=1$.

We have an exact sequence

$$
0 \rightarrow U \otimes \mathcal{O}_{X} \rightarrow \Theta_{X} \rightarrow V \otimes \mathcal{O}_{X} \rightarrow 0
$$

whose extension class is provided by $B^{\prime \prime}$, yielding a cohomology exact sequence $(* *) V \rightarrow U \otimes H^{1}\left(X, \mathcal{O}_{X}\right) \rightarrow H^{1}\left(X, \Theta_{X}\right) \rightarrow V \otimes H^{1}\left(X, \mathcal{O}_{X}\right) \rightarrow U \otimes H^{2}\left(X, \mathcal{O}_{X}\right)$, and the above cited corollary says that the first and the last maps are given by cup product and contraction with $B^{\prime \prime}$.

The following proposition allows us to describe the cohomology groups $H^{1}\left(X, \mathcal{O}_{X}\right), H^{2}\left(X, \mathcal{O}_{X}\right)$.

Proposition 4.5. Cat04 The Leray spectral sequence for the sheaf $\mathcal{O}_{X}$ and for the map $f: X \rightarrow Y$ is a spectral sequence which degenerates at the $E_{3}$ level and with $E_{2}$ term $=\left(H^{i}\left(\mathcal{R}^{j} f_{*} \mathcal{O}_{X}\right), d_{2}\right)$, where $d_{2}:\left(H^{i}\left(\mathcal{R}^{j} f_{*} \mathcal{O}_{X}\right)=\right.$ $H^{i}\left(\Lambda^{j}\left(\bar{U}^{\vee}\right) \otimes \mathcal{O}_{Y}\right)=\Lambda^{i}\left(\bar{V}^{\vee}\right) \otimes \Lambda^{j}\left(\bar{U}^{\vee}\right) \rightarrow \Lambda^{i+2}\left(\bar{V}^{\vee}\right) \otimes \Lambda^{j-1}\left(\bar{U}^{\vee}\right)$ is provided by cup product and contraction with $\bar{B}^{\prime} \in \Lambda^{2}\left(\bar{V}^{\vee}\right) \otimes(\bar{U})$.

The Leray spectral sequence yields then an exact sequence

$$
\begin{gathered}
0 \rightarrow H^{1}\left(Y, \mathcal{O}_{Y}\right)=\bar{V}^{\vee} \rightarrow H^{1}\left(X, \mathcal{O}_{X}\right) \rightarrow H^{0}\left(\mathcal{R}^{1} f_{*} \mathcal{O}_{X}\right)=\bar{U}^{\vee} \rightarrow \\
\rightarrow H^{2}\left(Y, \mathcal{O}_{Y}\right)=\Lambda^{2}\left(\bar{V}^{\vee}\right) \rightarrow H^{2}\left(X, \mathcal{O}_{X}\right),
\end{gathered}
$$

and the map

$$
H^{0}\left(\mathcal{R}^{1} f_{*} \mathcal{O}_{X}\right)=\bar{U}^{\vee} \rightarrow H^{2}\left(Y, \mathcal{O}_{Y}\right)=\Lambda^{2}\left(\bar{V}^{\vee}\right)
$$

is given by $\overline{B^{\prime}}$.

So we see again that $H^{1}\left(X, \mathcal{O}_{X}\right) \cong \bar{V}^{\vee} \oplus \operatorname{Ker}\left(\overline{B^{\prime}}\right)$, where we view $\overline{B^{\prime}}$ as a $\operatorname{map} \overline{B^{\prime}}: \bar{U}^{\vee} \rightarrow \Lambda^{2}\left(\bar{V}^{\vee}\right)$.

Thus, to determine $H^{1}\left(X, \Theta_{X}\right)$, it remains to compute $H^{2}\left(X, \mathcal{O}_{X}\right)$.

Since the Leray spectral sequence degenerates at $E_{3}$ level, we have

$$
H^{2}\left(X, \mathcal{O}_{X}\right) \cong E_{3}^{0,2} \oplus E_{3}^{2,0} \oplus E_{3}^{1,1},
$$

where $E_{3}^{0,2}=\operatorname{Ker}\left(d_{2}: E_{2}^{0,2} \rightarrow E_{2}^{2,1}\right)=\operatorname{Ker}\left({\overline{B^{\prime}}}^{2}: \Lambda^{2}\left(\bar{U}^{\vee}\right) \rightarrow \bar{U}^{\vee} \otimes \Lambda^{2}\left(\bar{V}^{\vee}\right)\right)$,

$$
\begin{aligned}
& E_{3}^{1,1}=\operatorname{Ker}\left(d_{2}: E_{2}^{1,1} \rightarrow E_{2}^{3,0}\right)=\operatorname{Ker}\left({\overline{B^{\prime}}}^{\prime}: \bar{U}^{\vee} \otimes \bar{V}^{\vee} \rightarrow \Lambda^{3}\left(\bar{V}^{\vee}\right)\right), \\
& E_{3}^{2,0}=\frac{E_{2}^{2,0}}{\operatorname{Im}\left(d_{2}: E_{2}^{0,1} \rightarrow E_{2}^{2,0}\right)}=\operatorname{Coker}\left(\overline{B^{\prime}}: \bar{U}^{\vee} \rightarrow \Lambda^{2}\left(\bar{V}^{\vee}\right)\right) .
\end{aligned}
$$

Now we want to determine the kernel of the coboundary map

$$
F: V \otimes H^{1}\left(X, \mathcal{O}_{X}\right) \rightarrow U \otimes H^{2}\left(X, \mathcal{O}_{X}\right),
$$

in the exact sequence $(* *)$

$$
V \rightarrow U \otimes H^{1}\left(X, \mathcal{O}_{X}\right) \rightarrow H^{1}\left(X, \Theta_{X}\right) \rightarrow V \otimes H^{1}\left(X, \mathcal{O}_{X}\right) \rightarrow U \otimes H^{2}\left(X, \mathcal{O}_{X}\right)
$$

in terms of the isomorphism

$$
V \otimes H^{1}\left(X, \mathcal{O}_{X}\right) \cong V \otimes\left(\bar{V}^{\vee} \oplus \operatorname{Ker}\left(\overline{B^{\prime}}\right)\right)=\left(V \otimes \bar{V}^{\vee}\right) \oplus\left(V \otimes \operatorname{Ker}\left(\overline{B^{\prime}}\right)\right),
$$


Lemma 4.6. The kernel of the coboundary map

$$
F: V \otimes H^{1}\left(X, \mathcal{O}_{X}\right) \rightarrow U \otimes H^{2}\left(X, \mathcal{O}_{X}\right),
$$

in the exact sequence $(* *)$ consists of the pairs $\left(\bar{L}, \sum_{i} v_{i} \otimes{\overline{u_{i}}}^{\vee}\right)$ such that

1) there exists $\bar{M} \in \bar{U}^{\vee} \otimes U$ such that $B^{\prime \prime}(\bar{L})=\bar{M} \circ \overline{B^{\prime}}: \Lambda^{2}(\bar{V}) \rightarrow U$,

2) $\sum_{i} B^{\prime \prime}\left(v_{i}\right) \otimes{\overline{u_{i}}}^{\vee}=0$ in $U \otimes \bar{V}^{\vee} \otimes \bar{U}^{\vee}$ for each choice of $v_{i} \in V,{\overline{u_{i}}}^{\vee} \in$ $\operatorname{Ker}\left(\overline{B^{\prime}}\right)$

\section{Proof.}

Since $F$ is given by cup product and contraction with $B^{\prime \prime}$, for $\bar{L} \in V \otimes \bar{V}^{\vee}$, $v \in V, \bar{u}^{\vee} \in \operatorname{Ker}\left(\overline{B^{\prime}}\right)$, we have

$$
F\left(\bar{L}, v \otimes \bar{u}^{\vee}\right)=\left(\zeta(\bar{L}), B^{\prime \prime}(v) \otimes \bar{u}^{\vee}\right),
$$

where $\zeta(\bar{L})=\left[B^{\prime \prime}(\bar{L})\right] \in \frac{\Lambda^{2}\left(\bar{V}^{\vee}\right) \otimes U}{\operatorname{Im}\left(\bar{B}^{\prime}\right) \otimes U}=E_{3}^{2,0} \otimes U$,

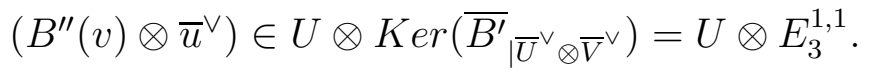

So $\zeta(\bar{L})=0$ if and only if $B^{\prime \prime}(\bar{L}) \in \operatorname{Im}\left(\overline{B^{\prime}} \otimes I d: \bar{U}^{\vee} \otimes U \rightarrow \Lambda^{2}\left(\bar{V}^{\vee}\right) \otimes U\right)$, i.e. there exists $\bar{M} \in \bar{U}^{\vee} \otimes U$ such that $B^{\prime \prime}(\bar{L})=\bar{M} \circ \overline{B^{\prime}}: \Lambda^{2}(\bar{V}) \rightarrow \bar{U} \rightarrow U$.

Q.E.D.

Remark 4.7. Observe that condition 1) says exactly that the pair $(L, M)$ belongs to the tangent space to the Appell Humbert space.

Proposition 4.8. The Kodaira Spencer map of the complete Appell Humbert family is surjective under one of the following assumptions:

i] $h^{1}\left(\mathcal{O}_{X}\right)=h^{1}\left(\mathcal{O}_{Y}\right)$, i.e., $\operatorname{Ker}\left(\overline{B^{\prime}}\right)=0$

ii] $d=1$ and $B^{\prime} \neq 0$

iii] $d=1$ and $B(v, x)$ non degenerate in the first factor.

iv] $X$ is parallelizable and $A$ is surjective as a linear map between $\Lambda^{2}(V \oplus$ $\bar{V}) \rightarrow(U \oplus \bar{U})$.

\section{Proof.}

By the previous remark and by lemma 3.13 the image of $\rho(L, M, \phi)$ inside $H^{1}\left(V \otimes \mathcal{O}_{X}\right)$ is simply the pair $(\bar{L}, 0)$.

Thus, we first observe that the subspace of the tangent vectors with $L=0$, namely, $\left\{(0, M, \phi) \mid M \circ B^{\prime}=0\right\}$ maps onto $\left\{(\bar{M}, \phi) \mid \bar{M} \circ \overline{B^{\prime}}=0\right\}=(U \otimes$ $\left.\operatorname{ker}\left(\overline{B^{\prime}}\right)\right) \oplus\left(U \otimes \bar{V}^{\vee}\right)=H^{1}\left(U \otimes \mathcal{O}_{X}\right)$.

Second, by condition 1) above, the image of the Kodaira Spencer map of the complete Appell Humbert family is surjective onto $\operatorname{ker}(F) \subset H^{1}\left(V \otimes \mathcal{O}_{X}\right)$ if and only if $\operatorname{ker}(F) \subset\left(V \otimes \bar{V}^{\vee}\right)$.

Therefore, we want that $\sum_{i} B^{\prime \prime}\left(v_{i}\right) \otimes{\overline{u_{i}}}^{\vee}=0$ in $U \otimes \bar{V}^{\vee} \otimes \bar{U}^{\vee}$ with $v_{i} \in V$, $\bar{u}_{i}^{\vee} \in \operatorname{Ker}\left(\overline{B^{\prime}}\right)$ implies $\sum_{i} v_{i} \otimes{\overline{u_{i}}}^{\vee}=0$.

Therefore this holds trivially under condition i], that $\operatorname{Ker}\left(\overline{B^{\prime}}\right)=0$.

In turn, condition ii] : $d=1$ and $B^{\prime} \neq 0$ implies condition i]. 
In the case $d=1$ we do not need to take a sum of simple tensors, but just one, and then $B^{\prime \prime}(v) \otimes \bar{u}^{\vee}=0$, for $\bar{u}^{\vee} \neq 0$, holds iff $B^{\prime \prime}(v)=0 \in\left(\bar{V}^{\vee} \otimes U\right)$. However, since we may assume $B^{\prime}=0$, the last condition is equivalent to $B(v)=0$, which contradicts iii].

Finally, we show that iv] implies i]: in fact we know that parallelizability is equivalent to the vanishing of $B^{\prime \prime}$. Whence, we have $A=B^{\prime} \oplus \overline{B^{\prime}}$, thus $A$ is surjective if and only if $B^{\prime}$ is surjective as a linear map $\Lambda^{2}(V) \rightarrow(U)$. But then the conjugate of the transpose is injective, i.e., condition i] holds.

Q.E.D.

Remark 4.9. On a compact complex manifold $X$ one has the Kodaira inequalities

$$
2 h^{1}\left(X, \mathcal{O}_{X}\right) \geq h^{1}\left(X, \mathcal{O}_{X}\right)+h^{0}\left(d \mathcal{O}_{X}\right) \geq b_{1}(X),
$$

which hold iff $X$ has a very good (cf. e.g. Cat04) Albanese variety, of dimension $h^{1}\left(X, \mathcal{O}_{X}\right)=h^{0}\left(d \mathcal{O}_{X}\right)=\frac{1}{2} b_{1}(X)$.

For principal holomorphic torus bundles then $h^{1}\left(\mathcal{O}_{X}\right)=h^{1}\left(\mathcal{O}_{Y}\right)$ implies by semicontinuity that every small deformation $X_{t}$ deforms together with an Albanese map $X_{t} \rightarrow Y_{t}$. If $d=1$ necessarily $X_{t} \rightarrow Y_{t}$ is an elliptic bundle, but for $d \geq 2$ we only have a differentiable torus bundle as a deformation, and therefore i] of the previous proposition yields a further contribution.

Recall the following

Theorem 4.10. Cat04 Let $f: X \rightarrow Y$ be a holomorphic principal bundle with base a complex torus $Y_{V}$ of dimension $m$, and fibre an elliptic curve $T_{U}$.

Assume moreover $\pi_{1}(X):=\Pi$ to be a nontrivial central extension

$$
1 \rightarrow \Lambda \rightarrow \Pi \rightarrow \Gamma \rightarrow 1
$$

classified by a cohomology class $\epsilon \neq 0 \in H^{2}(Y, \Lambda)$ whose associated bilinear form $A$ has an image of dimension $=2$.

Then every limit of manifolds in the complete Appell-Humbert family is again a holomorphic principal bundle $f^{\prime}: X^{\prime} \rightarrow Y^{\prime}$ with fibre an elliptic curve $T^{\prime}$, and thus occurs in the complete Appell-Humbert family .

The above result combines with the following one:

Theorem 4.11. Let $f: X \rightarrow Y$ be a holomorphic principal bundle with base a complex torus $Y:=Y_{V}$ of dimension $m=2$, and fibre an elliptic curve $T:=T_{U}$.

Then the complete Appell Humbert family is versal at $X$ if either $B^{\prime} \neq 0$, or $B^{\prime}=0$ and $B(v, x)$ non degenerate in the first factor, or $X$ is parallelizable and $A \neq 0$.

Proof. By Proposition 3.11 the complete Appell Humbert space is smooth for $m=2, d=1$, thus versality would follow from the surjectivity of the Kodaira Spencer map.

Surjectivity under the first two respective assumptions follows directly from ii] and iii] of proposition 4.8, whereas, if $X$ is parallelizable, we argue as in iv] 
of 4.8. $B^{\prime \prime}=0$, and if also $B^{\prime}=0$, then $A=0$, a contradiction. Thus $B^{\prime} \neq 0$ and we are done.

$$
\text { Q.E.D. }
$$

Proposition 4.12. Assume $m=2, d=1$ and assume further that $A$ is non degenerate. Then the case : there is $(U, V)$ such that $B^{\prime}=0$ and $B(v, x)$ is degenerate in the first factor occurs only if the associated pencil

$\mathbb{P}^{1} \cong \mathbb{P}\left((\Lambda \otimes \mathbb{R})^{\vee}\right) \hookrightarrow \mathbb{P}\left(\Lambda^{2}(\Gamma \otimes \mathbb{R})^{\vee}\right) \cong \mathbb{P}\left(\Lambda^{2}\left(\mathbb{R}^{4}\right)\right)$ intersects the Klein Pfaffian quadric in two distinct complex conjugate points.

Proof. Assume that $v \in V$ is such that $B(v, x)=0$ for each $x$, and recall the assumption $B^{\prime}=0$. We can choose $w \in V$ such that $\{v, w\}$ is a basis of $V$.

We have $B^{\prime \prime}(v, \bar{v})=0=\overline{B^{\prime \prime}}(v, \bar{v}), B^{\prime \prime}(v, \bar{w})=0=\overline{B^{\prime \prime}}(w, \bar{v})$.

We set $B^{\prime \prime}(w, \bar{v}):=\zeta$ so that $\overline{B^{\prime \prime}}(v, \bar{w})=-\bar{\zeta}$, and observe that $\zeta \neq 0$, otherwise $v$ is in the kernel of $A$.

Up to a change of basis replacing $w$ by $w+\mu v$, we may assume that also $B^{\prime \prime}(w, \bar{w})=0=\overline{B^{\prime \prime}}(w, \bar{w})$.

We have found two real isotropic subspaces for $A$, whose complexifications are spanned by $\{v, \bar{v}\},\{w, \bar{w}\}$ respectively.

These two subspaces are spanned by $\{v \pm \bar{v}\},\{w \pm \bar{w}\}$ and we compute the corresponding values of $B^{\prime \prime}$ on pairs of such vectors. Setting $\epsilon_{1}, \epsilon_{2}= \pm 1$ we get

$$
\begin{aligned}
B^{\prime \prime}\left(v+\epsilon_{1} \bar{v}, w+\epsilon_{2} \bar{w}\right) & =-\epsilon_{1} \zeta \\
\overline{B^{\prime \prime}}\left(v+\epsilon_{1} \bar{v}, w+\epsilon_{2} \bar{w}\right) & =-\epsilon_{2} \bar{\zeta} .
\end{aligned}
$$

We observe at this point that $e_{1}:=\zeta+\bar{\zeta}, e_{2}:=\frac{1}{i}(\zeta-\bar{\zeta})$ give a real basis for $\Lambda \otimes \mathbb{R}$, whence in the basis $\{2 \operatorname{Re}(v), 2 \operatorname{Im}(v), 2 \operatorname{Re}(w), 2 \operatorname{Im}(w)\} A$ is given by a $2 \times 2$ block matrix with diagonal blocks equal to zero and with upper right block equal to:

$$
M=\left(\begin{array}{cc}
-e_{1} & -e_{2} \\
e_{2} & -e_{1}
\end{array}\right) \text {. }
$$

Therefore, the corresponding Pfaffian of $\mu_{1} A_{1}+\mu_{2} A_{2}$, if $A_{1}, A_{2}$ are the components of $A$ with respect to the basis $e_{1}, e_{2}$, equals $\mu_{1}^{2}+\mu_{2}^{2}$, and it has no real roots.

$$
\text { Q.E.D. }
$$

Combining the last three stated results follows rightaway the following

Theorem 4.13. Consider the family of holomorphic principal torus bundles $f: X \rightarrow Y$ with base a complex torus $Y_{V}$ of dimension 2, and with fibre an elliptic curve $T_{U}$, corresponding to a bilinear form $A$ such that

1) $A$ is non degenerate and $\operatorname{Im} A$ has dimension 2

2) the associated pencil

$\mathbb{P}^{1} \cong \mathbb{P}\left((\Lambda \otimes \mathbb{R})^{\vee}\right) \hookrightarrow \mathbb{P}\left(\Lambda^{2}(\Gamma \otimes \mathbb{R})^{\vee}\right) \cong \mathbb{P}\left(\Lambda^{2}\left(\mathbb{R}^{4}\right)\right)$ intersects the Klein Pfaffian quadric in at least one real point.

Then this family forms a connected component of the Teichmüller space. 
Remark 4.14. One example of an elliptic bundle over a 2-dimensional torus is the so called Iwasawa manifold (cf. [K-M71]). It is parallelizable, being the quotient of the complex Lie group $N$ of $3 \times 3$ upper trangular matrices with all eigenvalues equal to 1 ( $N \cong \mathbb{C}^{3}$ as a complex manifold) by the discrete cocompact subgroup $\Pi$ which is the subgroup of the matrices with entries in the subring $\Lambda \subset \mathbb{C}, \Lambda:=\mathbb{Z}[i]$.

Nakamura observed that the Kuranishi family of $X$ is smooth of dimension 6 , and there are small deformations which are not parallelizable. This follows by our result in greater generality.

In this case, the alternating form $A$ is gotten as the antisymmetrization of the product map $\mathbb{C} \times \mathbb{C} \rightarrow \mathbb{C}$, which induces $\Lambda \times \Lambda \rightarrow \Lambda$.

One can explicitly calculate the integral bilinear map A, and it turns out that there are indeed cases where $B^{\prime}=0$ and $B(v, x)$ is degenerate in the first factor.

In fact, one can verify that the corresponding pencil of alternating forms intersects the Pfaffian Klein quadric in two distinct complex conjugate points. Thus, there are manifolds where the Kodaira Spencer image of the complete Appell Humbert family is not surjective.

If the complete Appell Humbert family does not yield an open set in the Kuranishi space, then the following should happen: we have a $X$ for which $h^{1}\left(\mathcal{O}_{X}\right)=3, h^{0}\left(d \mathcal{O}_{X}\right)=2=h^{0}\left(\Omega_{X}^{1}\right)$. But there is a small deformation $X_{t}$ such that $h^{1}\left(\mathcal{O}_{X_{t}}\right)=3, h^{0}\left(d \mathcal{O}_{X_{t}}\right)=1$.

It is interesting to see whether this can happen, for instance if this could be related to the existence of a fibration of $X$ over an elliptic curve which deforms along, producing another component of the Kuranishi space.

We conclude by observing, as recalled to us by Marian Aprodu:

Remark 4.15. Let $f: X \rightarrow Y$ be a holomorphic principal bundle with base a complex torus $Y:=Y_{V}$ of dimension $m$, and fibre a torus $T:=T_{U}$, such that $A \neq 0$.

Then $X$ is not Kähler.

Indeed, this was proven by Blanchard in Bla56 more generally for holomorphic fibre bundles whose transgression $H^{1}(T, \mathbb{R}) \rightarrow H^{2}(Y, \mathbb{R})$ is nonzero ( cf. also [Hö93]).

\section{Acknowledgements.}

The authors wish to thank Marian Aprodu, resp. Alessandro Ghigi, for a useful conversation.

\section{REFERENCES}

[Apr] M. Aprodu, "An Appell-Humbert theorem for hyperelliptic surfaces", J. Math. Kyoto Univ. 38, no. 1 (1998), 101-121.

[BPV84] W. Barth, C. Peters and A. Van de Ven, "Compact Complex Surfaces", Ergebnisse der Mathematik und ihrer Grenzgebiete, 3.F, B. 4, Springer-Verlag, 1984. 
[Bla53] A. Blanchard, "Recherche de structures analytiques complexes sur certaines variétés", C.R. Acad.Sci., Paris 238 (1953), 657-659.

[Bla56] A. Blanchard, "Sur les variétés analytiques complexes", Ann.Sci. Ec. Norm. Super., III Ser., 73 (1956), 157-202.

[Cal58] E. Calabi, "Construction and properties of some 6-dimensional almost complex manifolds, Trans. Amer. Math.Soc. 87 (1958), 407-438.

[Cat91] F. Catanese, "Moduli and classification of irregular Kähler manifolds (and algebraic varieties) with Albanese general type fibrations. Appendix by Arnaud Beauville." Inv. Math. 104 (1991) 263-289; Appendix 289.

[Cat95] F. Catanese, "Compact complex manifolds bimeromorphic to tori." Proc. of the Conf. "Abelian Varieties", Egloffstein 1993, De Gruyter (1995), 55-62.

[Cat02] F. Catanese, "Deformation types of real and complex manifolds", Proc. of the ChenChow Memorial Conference, Contemporary trends in algebraic geometry and algebraic topology (Tianjin, 2000), Nankai Tracts Math., 5, World Sci. Publishing, River Edge, NJ (2002), 195-238 .

[Cat04] F. Catanese, "Deformation in the large of some complex manifolds, I', Ann. Mat. Pura Appl. (4) 183, no. 3, Volume in Memory of Fabio Bardelli, (2004), 261-289.

[Che58] S.S. Chern, "Complex manifolds", Publ. Mat. Univ. Recife (1958).

[FG65] W. Fischer, H. Grauert, "Lokal-triviale Familien kompakter komplexer Mannigfaltigkeiten" Nachr. Akad. Wiss. Göttingen, II. Math.-Phys. Kl. 1965 (1965), 89-94 .

[Hir62] H. Hironaka, "An example of a non-Kählerian complex-analytic deformation of Kählerian complex structures", Ann. Math. (2) 75, (1962),190-208.

[Hö93] T. Höfer, "Remarks on torus principal bundles", J. Math. Kyoto Univ. 33 , no. 1 (1993), 227-259.

[Ko64] K. Kodaira," On the structure of complex analytic surfaces I", Amer. J. Math. 86 (1964), 751-798 .

[Ko68] K. Kodaira ," On the structure of complex analytic surfaces IV", Amer. J. Math. 90 (1968), 1048-1066.

[K-M71] K. Kodaira, J. Morrow ,"Complex manifolds" Holt, Rinehart and Winston, New York-Montreal, Que.-London (1971).

[K-S58] K. Kodaira, D. Spencer "On deformations of complex analytic structures I-IT", Ann. of Math. 67 (1958), 328-466 .

[Ku1] M. Kuranishi, "On a type of family of complex structures", Ann. of Math. (2) 74 (1961), 262-328.

[Ku2] M. Kuranishi, "New proof for the existence of locally complete families of complex structures", Proc. Conf. Complex Analysis (Minneapolis, 1964) Springer, Berlin (1965), 142-154.

[Nak75] I. Nakamura, "Complex parallelisable manifolds and their small deformations" J. Differ. Geom. 10, (1975),85-112 .

[Nak98] I. Nakamura, "Global deformations of $\mathbb{P}^{2}$-bundles over $\mathbb{P}^{1}$ " J. Math. Kyoto Univ. 38, No.1 (1998), 29-54 .

[Somm75] A. J. Sommese, "Quaternionic manifolds", Math. Ann. 212 (1975), 191-214.

[Su70] T. Suwa, "On hyperelliptic surfaces", J. Fac. Sci. Univ. Tokyo, 16 (1970), 469-476

[Su75] T. Suwa, "Compact quotient spaces of $\mathbb{C}^{2}$ by affine transformation groups", J. Differ. Geom. 10, (1975) 239-252.

[Su77-I] T. Suwa, "Compact quotients of $\mathbb{C}^{3}$ by affine transformation groups, I.", Several complex Variables, Proc. Symp. Pure Math. 30, Part 1, Williamstown 1975, (1977), 293-295.

[Su77-II] T. Suwa, "Compact quotients of $\mathbb{C}^{3}$ by affine transformation groups, II.", Complex Analysis and Algebraic Geometry, Iwanami Shoten (1977), 259-278 .

[Ue75] K. Ueno, "Classification theory of algebraic varieties and compact complex spaces. Lecture Notes in Mathematics 439, Springer-Verlag, XIX, 278 p.(1975).

[Ue80] K. Ueno, "On three-dimensional compact complex manifolds with non positive Kodaira dimension", Proc. Japan Acad., vol.56,S.A.n.10 (1980), 479-483. 
[Ue82] K. Ueno, "Bimeromorphic Geometry of algebraic and analytic threefolds", in 'C.I.M.E. Algebraic Threefolds, Varenna 1981' Lecture Notes in math. 947 (1982), 1-34 .

[Ue87] K. Ueno, "On compact analytic Threefolds with non trivial Albanese Tori", Math. Ann. 278 (1987), 41-70.

\section{Author's address:}

Prof. Fabrizio Catanese

Lehrstuhl Mathematik VIII

Universität Bayreuth

D-95440, BAYREUTH, Germany

e-mail: Fabrizio.Catanese@uni-bayreuth.de

Dr. Paola Frediani

Dipartimento di Matematica

Università di Pavia

I-27100 Pavia, Italy

e-mail: paola.frediani@unipv.it 